\title{
Análisis del nivel de alfabetización en salud, en pacientes con insuficiencia renal crónica en hemodiálisis
}

\author{
Raquel Pelayo Alonso, Patricia Martínez Álvarez, Sara Merino García, Alicia Labrador Pérez, Soraya Sánchez \\ Cano, José Luis Cobo Sánchez
}

Unidad de Hemodiálisis del Hospital Universitario Marqués de Valdecilla. Santander. Cantabria. España.

\section{Resumen}

Introducción: La alfabetización en salud tiene un impacto significativo sobre la salud individual, más aún en pacientes de edad avanzada, enfermos crónicos 0 pacientes polimedicados, características que están presentes en el paciente en tratamiento con hemodiálisis. Un bajo nivel de alfabetización en salud se asocia peores resultados en salud.

Objetivo: Valorar el nivel de alfabetización en salud en pacientes con insuficiencia renal crónica en tratamiento con hemodiálisis y compararla con la población general y otra patología crónica (insuficiencia cardíaca).

Material y Método: Estudio descriptivo prospectivo en 37 pacientes sometidos a hemodiálisis crónica. Para valorar el nivel de alfabetización en salud se utilizó el cuestionario Health Literacy Survey-European Union (HLS-EU-Q).

Resultados: El promedio del índice general de alfabetización fue de $26,4 \pm 7,32$ puntos. Más del $83 \%$ de la muestra presentó un nivel de alfabetización inadecuado o problemático. Nuestra población presentó peores niveles de alfabetización comparados con pacientes con insuficiencia cardiaca (inadecuado o problemático $83,8 \%$ vs $79,6 \%$, p: NS) y con la población general (inadecuado o problemático $88,8 \%$ vs $58,3 \%, \mathrm{p}<0,002$ ).
Conclusiones: Los datos conseguidos reflejan un nivel de alfabetización problemática o inadecuada, lo que indica que los pacientes a los que atendemos tienen dificultades para interpretar y aplicar los mensajes educativos de los profesionales sanitarios.

PALABRAS CLAVE: alfabetización en salud; insuficiencia renal crónica; hemodiálisis.

Analysis of the level of health literacy in patients with chronic renal failure on hemodialysis

\section{Abstract}

Introduction: Health literacy has a significant impact on individual health, especially in elderly patients, chronic patients or polymedicated patients, characteristics present in hemodialysis patients. A low level of health literacy is associated with poorer health outcomes.

Aim: To assess the level of health literacy in patients with chronic renal failure on hemodialysis and to compare it with the general population and other chronic pathology (heart failure).

Material and Method: Descriptive prospective study in 37 patients on chronic hemodialysis. To assess the level of health literacy, the Health Literacy Survey-European Union (HLS-EU-Q) questionnaire was used.

Results: The average overall literacy rate was 26.4 \pm 7.32 points. More than $83 \%$ of the sample had an inadequate or problematic level of literacy. Our 
population had worse literacy levels compared to patients with heart failure (inadequate or problematic: $83.8 \%$ vs $79.6 \%$; p: NS) and with the general population (inadequate or problematic: $88.8 \%$ vs $58.3 \% ; p<0.002$ ).

Conclusions: The data obtained reflect a problematic or inadequate level of health literacy, which indicates that patients attended have difficulties interpreting and applying the educational messages of health professionals.

KEYWORDS: health literacy; chronic renal failure; hemodialysis.

\section{Introducción}

En los últimos años, el personal sanitario se está interesando cada vez más por la relación existente entre el conocimiento, habilidades y capacidades para cuidarse que poseen las personas a las que atienden y el nivel de salud que poseen ${ }^{1}$. Esto ha dado lugar a la aparición de términos como Alfabetización en Salud (AeS), traducido del vocablo inglés health literacy, término con gran repercusión en la comunidad científica.

Muchos son los autores que ha establecido su definición ${ }^{2}$, aunque la más conocida y empleada es la que recoge la Organización Mundial de la Salud, en su glosario de términos de salud pública, dónde se define como "las habilidades sociales y cognitivas que determinan el nivel de motivación y la capacidad de una persona para acceder, entender y utilizar la información de forma que le permita promover y mantener una buena salud"'3. En definitiva, la AeS no se refiere sólo a entender la información que da el profesional sanitario, sino que implica saber cómo acceder a información apropiada, juzgar e interpretar dicha información y ser capaz de aplicarla en función de las circunstancias y necesidades personales ${ }^{1}$.

La AeS tiene un impacto significativo sobre la salud individual, más aún en pacientes de edad avanzada, enfermos crónicos o pacientes polimedicados, características que están presentes en el paciente renal en tratamiento con hemodiálisis (HD) 4 .

Un bajo nivel de AeS se asocia con una comprensión insuficiente de conceptos, peor gestión de la enfermedad y de actividades de autocuidados, menor uso de sis- temas preventivos, errores en la toma de medicación y una mayor dificultad para entender instrucciones, lo que condiciona peores resultados en salud y por lo tanto un mayor coste social y económico. Debido a las características de los pacientes sometidos a HD y de la importancia de los autocuidados para el éxito de esta terapia, nos planteamos los objetivos de este estudio.

\section{Objetivo}

Valorar el nivel de AeS en pacientes con insuficiencia renal crónica (IRC) en tratamiento con HD y comparar dicho nivel de AeS con el de la población general y con el de otro paciente con patología crónica, como es la insuficiencia cardíaca.

\section{Material y Método}

Se realizó un estudio descriptivo prospectivo en 37 pacientes mayores de 18 años, sometidos a HD crónica, desde hacía más de un mes en la Unidad de Hemodiálisis del Hospital Universitario Marqués de Valdecilla. Se establecieron como criterios de exclusión el presentar una situación clínica que impidiese la cumplimentación del cuestionario, el presentar un deterioro cognitivo, una mala comprensión del idioma y la negativa del paciente a participar en el estudio tras recibir la información pertinente. Todos los pacientes firmaron el consentimiento informado antes del inicio de la recogida de datos.

Para valorar el nivel de AeS se utilizó el cuestionario Health Literacy Survey-European Union (HLSEU-Q $)^{5}$, en su versión española validada, que consta de 47 ítems sobre situaciones relacionadas sobre la gestión de la información, asistencia sanitaria, prevención de la enfermedad y promoción de la salud. El cuestionario fue autocumplimentado por el paciente, recibiendo ayuda del equipo investigador aquellos pacientes que así lo solicitaron. Cada ítem se responde en una escala tipo Likert de 4 puntos (de muy fácil, a muy difícil). Se obtiene un índice general de AeS cuyo valor máximo será 50 puntos y el mínimo será 0 puntos; y 3 subíndices secundarios (atención y cuidados de salud, prevención de enfermedades y promoción de la salud), con el mismo rango de puntuación posible. Según la puntuación alcanzada en el índice general de AeS, se establecen cuatro niveles de alfabetización: inadecuada (0-25 puntos); problemática (26-33 puntos); suficiente (34-42 puntos) y excelente (42-50 puntos). Los datos se analizaron según las normas de los autores. 
Además se recogieron datos sociodemográficos y clínicos como el sexo, edad, comorbilidad asociada (calculada mediante el índice de Charlson), tiempo en tratamiento renal sustitutivo, número de ingresos hospitalarios y visitas a Servicio de Urgencias en el último año, estado civil, convivencia, nivel de ingresos mensuales, nivel educativo y autopercepción del estado de salud.

Para comparar los resultados con población general española se tomaron como referencia los obtenidos por Sorensen et $\mathrm{al}^{6}$ y con pacientes con insuficiencia cardíaca crónica los publicados en el estudio de Santesmases y cols ${ }^{7}$. En ambos estudios utilizaron como instrumento para evaluar la AeS el HLS-EU-Q.

Para el análisis de los datos se utilizó los paquetes estadísticos IBM SPSS Statistics v.20.0 y EpiDat v.4.2. Se realizó un análisis descriptivo de las variables a estudio, utilizando medidas de tendencia central en las variables cuantitativas, y frecuencias y porcentajes para las variables categóricas. Se realizaron análisis bivariantes entre el índice general de AeS y distintas características de los pacientes (sexo, edad - según cuartiles de la población a estudio-, nivel de estudios, autopercepción del estado de salud, número de visitas a urgencias en el último año, número de ingresos hospitalarios en el último año, estado civil, tipo de convivencia, ingresos mensuales y tiempo en TRS - según cuartiles de la población a estudio-), mediante el test de ANOVA o el coeficiente de correlación de Pearson; y con las puntuaciones del índice general de AeS de la población general y de la población con insuficiencia cardiaca mediante el Z-test. Todas las pruebas estadísticas utilizaron un nivel de alfa $<0,05$ y el intervalo de confianza del $95 \%$.

\section{Resultados}

Se estudiaron 37 pacientes con una edad media alrededor de los 64 años siendo mayoritariamente hombres, con un tiempo medio en TRS de unos 5 años. En la tabla 1 se muestran las características de la población de estudio.

El promedio del índice general de AeS fue de $26,4 \pm 7,32$ puntos. Los resultados de los subíndices se muestran en la tabla 2. Según los niveles de alfabetización estable-
Tabla 1. Características de los participantes en el estudio.

\begin{tabular}{|c|c|c|}
\hline Característica & & $\mathbf{N}=37(\%)$ \\
\hline Sexo & $\begin{array}{l}\text { Hombre } \\
\text { Mujer }\end{array}$ & $\begin{array}{l}20(54,1 \%) \\
17(45,9 \%)\end{array}$ \\
\hline Edad & (Media \pm DE) & $64,51 \pm 16,57$ años \\
\hline $\begin{array}{l}\text { Tiempo en tratamiento } \\
\text { renal sustitutivo }\end{array}$ & (Media $\pm \mathrm{DE}$ ) & $63,51 \pm 45$ meses \\
\hline Índice de Comorbilidad & (Media $\pm \mathrm{DE}$ ) & $4,7 \pm 5$ puntos \\
\hline Estado de Salud & $\begin{array}{l}\text { Bueno } \\
\text { Regular } \\
\text { Malo } \\
\text { Muy Malo }\end{array}$ & $\begin{array}{l}12(32,4 \%) \\
15(40,5 \%) \\
9(24,3 \%) \\
1(2,7 \%)\end{array}$ \\
\hline Urgencias / año & $\begin{array}{l}\text { Ninguna } \\
\text { Menos de } 3 \\
3 \text { o más }\end{array}$ & $\begin{array}{l}19(51,4 \%) \\
15(40,5 \%) \\
3(8,1 \%)\end{array}$ \\
\hline Ingresos hospitalarios /año & $\begin{array}{l}\text { Ninguno } \\
\text { Menos de } 3 \\
3 \text { o más }\end{array}$ & $\begin{array}{l}18(48,6 \%) \\
18(48,6 \%) \\
1(2,7 \%)\end{array}$ \\
\hline Estado civil & $\begin{array}{l}\text { Soltero } \\
\text { Casado / En pareja } \\
\text { Divorciado / separado } \\
\text { Viudo }\end{array}$ & $\begin{array}{l}6(16,2 \%) \\
23(62,2 \%) \\
4(10,8 \%) \\
4(10,8 \%)\end{array}$ \\
\hline Convivencia & $\begin{array}{l}\text { Solo } \\
\text { Pareja } \\
\text { Hijos / otros familiares }\end{array}$ & $\begin{array}{l}5(13,5 \%) \\
12(32,4 \%) \\
20(54 \%)\end{array}$ \\
\hline Ingresos mensuales & $\begin{array}{l}\text { Menos de } 500 € \\
\text { Entre } 500 \text { y } 1000 € \\
\text { Entre } 1001 \text { y } 1500 € \\
\text { Más de } 1500 €\end{array}$ & $\begin{array}{l}4(10,8 \%) \\
12(32,4 \%) \\
10(27 \%) \\
11(29,7 \%)\end{array}$ \\
\hline Nivel de estudios & $\begin{array}{l}\text { Sin estudios } \\
\text { Estudios primarios } \\
\text { Estudios secundarios o FP } \\
\text { Estudios universitarios }\end{array}$ & $\begin{array}{l}1(2,7 \%) \\
14(37,8 \%) \\
17(45,9 \%) \\
5(13,5 \%)\end{array}$ \\
\hline
\end{tabular}

DE: Desviación típica.

Tabla 2. Resultado del Índice general de Alfabetización en Salud y de subíndices.

\begin{tabular}{|c|c|c|}
\hline & Media \pm DE & DE \\
\hline Índice general de AeS & 26,4 & 7,32 \\
\hline \multicolumn{3}{|l|}{ Subíndices } \\
\hline - Atención y cuidados de salud & 27,66 & 8,04 \\
\hline - Prevención de enfermedades & 26,94 & 8,6 \\
\hline - Promoción de la salud & 24,4 & 7,6 \\
\hline
\end{tabular}

AeS: Alfabetización en Salud. DE: Desviación típica.

cidos en función de la puntuación alcanzada en el índice general, más del $83 \%(n=31)$ presentó un nivel de AeS inadecuado o problemático (figura 1). Los grupos con menor AeS fueron los hombres, pacientes con edad $>77$ años; aquellos con un bajo nivel académico y pacientes que viven con otros familiares que no son la pareja. 


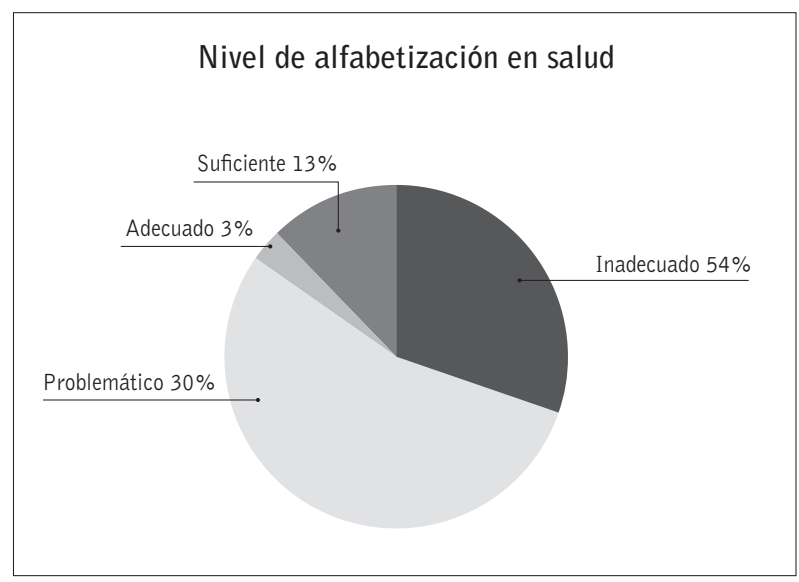

Figura 1. Distribución del nivel de alfabetización en función de la puntuación obtenida en el índice general de AeS.
En la tabla 3 se muestran los resultados del análisis ANOVA entre el índice general de alfabetización y diferentes variables de estudio.

Nuestra población presentó peores niveles de AeS comparados con pacientes con insuficiencia cardiaca (inadecuado o problemático $83,8 \%$ vs $79,6 \%$, p: NS) y con la población general (inadecuado o problemático $88,8 \%$ vs $58,3 \%, p<0,002$ ).

\section{Discusión}

El nivel de AeS de los pacientes con IRC sometidos a HD que se dializan en nuestro centro indica que estos pacientes presentan dificultades para comprender e interpretar la información referente a su estado de salud y a los cuidados que precisan. Estudios previos ${ }^{8-10}$ ya reflejan un alto porcentaje de una inadecuada alfabetización para el paciente con insuficiencia renal, no distinguiendo pacientes en estadios tempranos 0 avanzados de la enfermedad o con distintas opciones terapéuticas, ya sean, técnicas dialíticas 0 trasplante renal. Sin olvidar, que los instrumentos de medida, difieren de una investigación a otra pudiendo dar resultados no comparables.

El concepto de AeS está basado en un constructo multidimensional, que se ve afectado por distintos determinantes de la salud, tanto macro (socio-demográficos, psicosociales, culturales), como individuales (características individuales, alfabetización en general, experiencias personales previas durante procesos de salud y su interacción con el sistema sanitario) $)^{11-12}$.

En este estudio se ha utilizado como instrumento de medida el HLS-EU-Q ${ }^{5}$, cuestionario desarrollado por el Consorcio Europeo de AeS para medir y analizar la AeS de la población europea, habiendo sido utilizado por ocho países europeos, entre ellos, España. 
El índice de AeS de nuestra muestra, es muy inferior al que presenta la población general española. Resultados muy esperables ya que las características sociodemográficas de ambas poblaciones no son las mismas. En el estudio europeo de referencia ${ }^{6}$, la edad media de la población española es de 48 años, y un $21 \%$ del total, son mayores de 65 años, mientras que en nuestra muestra, la edad media supera los 64 años y los mayores de 65 años supera el $54 \%$ del total. Otro factor importante que difiere ambas muestras y que por lo tanto puede incidir en las diferencias de los resultados de AeS, es la existencia de una enfermedad de larga duración, no estando presente en el $66,4 \%$ de la población española.

La bibliografía refiere que la AeS tiene una implicación directa sobre pacientes de edad avanzadas, pacientes crónicos o polimedicados ${ }^{3,4}$, de ahí que quisiéramos comparar nuestros resultados con otra patología crónica. El estudio de Santesmases y $\mathrm{col}^{7}$ establece un índice bajo de AeS igual que en nuestra muestra, aunque con un menor nivel de alfabetización problemática. Un aspecto a tener en cuenta y que pudiera interferir es el ámbito en el que se realizan ambos estudios, Atención Primaria frente a una Unidad Hospitalaria.

Al igual que en nuestra muestra, aquellos pacientes con mayor edad presentan niveles más bajos de $\mathrm{AeS}^{7}$. Ese aspecto debe ser tenido en cuenta por los profesionales sanitarios. Algunos estudios ${ }^{2}$ sugieren que esto puede ser atribuible a la pérdida de habilidades cognitivas que lleva asociado el envejecimiento y que resultan necesarias para el procesamiento de la información.

Otro aspecto que también se relaciona con un bajo nivel de AeS es el nivel académico. Tanto nuestros datos, como los del estudio de Santesmases y $\mathrm{col}^{7}$ y del estudio europeo de referencia ${ }^{6}$, sugieren que la población con menos nivel educativo presentan una mayor dificultad para comprender la información necesaria para lograr un manejo adecuado de su enfermedad.

Una variable que no se ha tenido en cuenta en este estudio ha sido el valorar la adherencia al tratamiento, incluyendo medidas de autocuidado incluyendo dieta e ingesta hídrica, factores de gran importancia en esta fase de la enfermedad para el paciente renal en HD.

Podemos concluir diciendo que los datos obtenidos reflejan un nivel de AeS problemática o inadecuada, lo que indica que los pacientes a los que atendemos tienen dificultades para interpretar y aplicar los mensajes educativos de los profesionales sanitarios.
Nuestra población presenta peores niveles de AeS que la población general y que pacientes con otras patologías crónicas, como la insuficiencia cardíaca.

Es necesario que se adapten los programas de educación para la salud, incluyendo materiales informativos que capaciten al paciente a tomar decisiones informadas sobre su autocuidado, mejorando por lo tanto, la alfabetización en salud ${ }^{13-17}$.

Recibido: 12 julio 2017

Revisado: 25 julio 2017

Modificado: 3 agosto 2017

Aceptado: 10 agosto 2017

\section{Bibliografía}

1. Navarro MD, Rudd R, Rosenfeld L, Arrighi E. Alfabetización en salud. Implicación en salud: implicación en el sistema sanitario. Med Clin. 2016; 147(4):171-175.

2. Luna A. La alfabetización en salud de la población española: Variables relacionadas según los resultados del proyecto europeo de alfabetización en salud [Tesis doctoral]. Murcia: Área de Medicina Legal y Forense de la Facultad de Medicina, Universidad de Murcia; 2015.

3. Falcón M, Luna A. Alfabetización en salud: concepto y dimensiones. Proyecto europeo de alfabetización en salud. RCyS. 2012;2:91-98.

4. Costa G, Moreso F, Cantarell MC, Seron D. Alfabetización en salud y enfermedad renal crónica. Nefrología. 2017;37(2):115-117.

5. Sorensen K, Van den Broucke S, Pelikan J, Fullam J, Doyle G, Slonska $S$ et al. Measuring health literacy in populations: illuminating the desgin and development process of the Eupean Health Literacy Survey Questionnaire (HLS-EU-Q). BMC Public Health. 2013;13:948.

6. Sorensen K, Pelikan J, Röthlin F, Ganahl K, Slonska Z, Doyle G et al. Health literacy in Europe: comparative results of the European health literacy survey (HLS-EU). Eur J Public Health. 2015;25(6):1053-1058. 
7. Santesmases R, González L, Real J, Borrás A, Sisó A, Dolors MA. Alfabetización en salud en pacientes con insuficiencia cardiaca atendidos en atención primaria. Aten Primaria. 2017;49(1)28-34.

8. Ann B. Health literacy in nephrology: why is important?. Am J Kidney Dis. 2013;62(1):3-6

9. Devraj R, Borrego M, Vilay AM, Gordon EJ, Pailden J, Horowitz B. Relationship between health literacy and kidney function. Neprhology (Carlton). 2015;20:360-7. Consultado en: http:// dx.doi.org/10.1111/nep.12425.

10. Lambert K, Mullan J, Mansfield K, Lonergan M. A cross-sectional comparison of health literacy déficits among patients with chronic disease. J Health Commun. 2015;20:16-23.

11. Altin SV, Finke I, Kautz-Freimuth S, Stock S. The evolution of health literacy assessment tools: a systematic review. BMC Public Health. 2014;14:1207.

12. Cabellos-García AC, Castro-Sánchez E, MartínezSabater A, Gea-Caballero VA. Influencia de la alfabetización en salud sobre la anticoagulación oral: un factor aún desconocido. Aten Primaria. 2017. Disponible en: http://dx.doi.org/10.1016/j. aprim.2017.03.012
13. López-Dicastillo 0, Canga-Armayor N, Mujika A, Pardavila-Belio MI, Belintxon M, Serrano-Monzó I, Pumar-Méndez MJ. Cinco paradojas de la promoción de la salud. Gac Sanit. 2017;31(3):269272.

14. Rudd RE, Groene OR, Navarro-Rubio MD. On health literacy and health outcomes: background, impact, and future directions. Rev Calid Asist. 2013;28(3):188-92.

15. Lopez-Vargas PA, Tong A, Howell M, Craig JC. Educational Interventions for Patients With CKD: A Systematic Review. Am J Kidney Dis. 2016;68(3):353-70.

16. Skelton $S L$, Waterman AD, Davis LA, Peipert JD, Fish AF. Applying best practices to designing patient education for patients with end-stage renal disease pursuing kidney transplant. Prog Transplant. 2015;25(1):77-84.

17. Cavanaugh KL, Wright Nunes JA. Establishing Evidence for the Future of Kidney Disease Education. Am J Kidney Dis. 2016;68(3):335-7. 